\title{
Hommage à Luc Saïdj
}

Luc Saïdj vient, hélas, de nous quitter, le 5 novembre dernier. $C^{\prime}$ est avec émotion que nous rédigeons ces quelques lignes destinées à lui rendre hommage car Luc n'était pas seulement un collègue admiré et respecté, il était aussi un véritable ami, et d'une rare fidélité.

Le Doyen Saïdj a eu un itinéraire et une carrière exemplaires à de multiples égards. Son parcours, tout d'abord, montre toute sa détermination et son énergie. Né en 1943 en Algérie

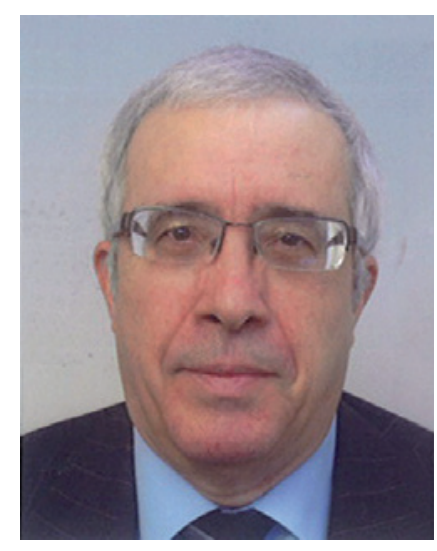
à Tizi-Ouzou, venu ensuite étudier à Lyon, Luc Saïdj a peu à peu gravi tous les échelons : étudiant à I'IEP, puis à la Faculté de droit, titulaire de deux diplômes d'études supérieures, il a soutenu en 1972 son doctorat d'État en droit (La notion de territoire en droit public français contemporain sous la direction de René Pelloux). Il fut successivement assistant, chargé de cours, maître-assistant et, à partir de 1989, professeur des universités, donnant à sa carrière une orientation de plus en plus tournée vers les finances publiques.

Et quelle carrière! Des trois fonctions attachées à notre métier d'universitaire, non seulement il ne négligea aucune, mais il les exerça chacune de façon remarquable.

En premier lieu, nous gardons le souvenir d'un enseignant qui rendait lumineuses toutes les disciplines du droit public, depuis les premières années de Licence jusqu'à la 5e année. II s'est progressivement spécialisé en finances publiques et en comptabilité publique, matières réputées arides, où il n'est pas évident d'intéresser de larges amphis : mais ses cours vous donnaient tous les éléments nécessaires, tout était expliqué avec rigueur et clarté, rien n'était omis...

En deuxième lieu, Luc Saïdj était un chercheur infatigable (et jusqu'à ces derniers mois...) : combien de thèses dirigées, de contributions à des colloques, d'épais fascicules de Jurisclasseurs, d'articles (notamment dans cette revue), d'ouvrages rédigés... Une abondante production scientifique va marquer toute sa carrière avec en particulier le manuel de Finances publiques (Dalloz) dont la première édition sera publiée en 1995. II rédigera en 2017 un avantpropos à l'occasion de la dixième édition de ce manuel.

Il a longtemps participé à l'animation de la Société française de finances publiques (SFFP) dont il fut le secrétaire général. II a été le promoteur de champs d'études en général négligés par les universitaires : ainsi de la comptabilité publique, notamment. Ceci explique qu'il ait été nommé au Comité consultatif d'orientation du Conseil de normalisation des comptes publics (CNOCP).

Mais on le retrouve œuvrant dans bien d'autres domaines et en particulier au comité technique radiophonique et au comité territorial de l'audiovisuel à Lyon.

Enfin, l'institution universitaire, et tout spécialement la Faculté de droit de l'Université Lyon 3, lui doit beaucoup : Luc Saïdj fut tout à la fois, ou successivement, directeur de DEA, de Centres ou d'Équipe de recherche (et notamment du Centre d'Études et de Recherches Financières et Fiscales - CERFF), mais aussi Doyen de la Faculté de droit, et Vice-président de l'Université Lyon 3 (bien entendu aux finances...). II fut, notamment, un Doyen exemplaire, entreprenant, innovant, juste et proche de chacun, d'une exceptionnelle probité intellectuelle, et profondément respecté. 
Mais il a rayonné bien au-delà de l'institution lyonnaise et même du cadre hexagonal, puisqu'il fut très souvent invité pour des cours, des conférences, des soutenances de thèse dans de multiples universités étrangères: Algérie (qu'il n'a jamais oubliée), Cameroun, Laos, Liban, Roumanie, Sénégal, Tunisie, Canada, Viet-Nam, Cambodge, Algérie, Bélarus, Ukraine, Pologne, Egypte, Chine... et la liste n'est pas exhaustive! Ce foisonnement d'activités se double ainsi d'une très grande sensibilité aux questions de coopération internationale; il sera d'ailleurs chargé des relations internationales à l'Université Lyon 3 de 1997 à 2007.

Les Mélanges qui lui ont été consacrés en 2012 en même temps qu'à ses collègues lyonnais, les professeurs Gabriel Montagnier et Jean-Pierre Lassale (Figures lyonnaises des finances publiques, L'Harmattan, coll. Finances publiques), sont un précieux témoignage de son itinéraire et de tout son apport à l'Université et au dehors, aux Finances publiques et plus globalement à la collectivité et à la communauté universitaire.

Au-delà de toutes ces grandes compétences, Luc Saïdj était d'une exceptionnelle humanité, toujours à l'écoute des autres (qu'ils soient collègues, doctorants, étudiants, personnel administratif, voisin...). Homme de culture, aimant le sport tout en appréciant aussi d'excellents repas, il était riche d'anecdotes, d'informations, de projets, de suggestions... mais aussi curieux et ouvert à tous. Être à ses côtés et collaborer avec lui pouvait aussi, c'est vrai, devenir redoutable lorsqu'on avait omis tel aspect ou mal compris tel élément : mais l'ignorance était alors balayée par un encouragement à aller de l'avant.

Luc nous a quittés, mais il reste et restera longtemps présent : par ses nombreux travaux, ses réflexions, ses idées, mais aussi par tous les souvenirs que nous gardons de lui, y compris dans les dernières années, à Limas, entouré de la protection bienveillante de Monique, sa femme, de ses nombreux chats et d'une admirable bibliothèque.

Jean-Luc ALBERT

Professeur des universités, Aix Marseille Université

Sylvie CAUDAL

Professeure émérite à l'Université Jean Moulin Lyon 3 